\title{
AVALIAÇÃO DE PROCEDIMENTOS PARA QUANTIFICAÇÃO ESPECTROFOTOMÉTRICA DE FLAVONOIDES TOTAIS EM FOLHAS DE Bauhinia forficata LINK
}

\author{
Graziella Silvestre Marques, Rebeka Priscila Maranhão Monteiro, Waleska de Figueirêdo Leão, Magaly Andreza Marques \\ Lyra, Monize Santos Peixoto, Pedro José Rolim-Neto, Haroudo Sátiro Xavier e Luiz Alberto de Lira Soares* \\ Departamento de Ciências Farmacêuticas, Universidade Federal de Pernambuco, Rua Arthur de Sá, s/n, 50740-521 Recife - PE, Brasil
}

Recebido em 11/5/11; aceito em 20/9/11; publicado na web em 6/12/11

\begin{abstract}
EVALUATION OF PROCEDURE FOR SPECTROPHOTOMETRIC QUANTIFICATION OF TOTAL FLAVONOIDS IN LEAVES OF Bauhinia forficata LINK. Interest in analytical methods for quality control of herbal drugs has grown sharply due to the scarcity of monographs in official manuals. Thus, the aim of the present study was to evaluate analytical procedures for quantitative determination of flavonoids from leaves of Bauhinia forficata Link (pata-de-vaca). Two procedures for quantification of total flavonoids (with and without acid hydrolysis) by spectrophotometry were tested. The proposed methods proved to be specific, sensitive, precise, accurate and robust, being suitable for routine laboratory use.
\end{abstract}

Keywords: Bauhinia forficata Link; flavonoids; UV-Vis spectrophotometric.

\section{INTRODUÇÃO}

A utilização de plantas medicinais e fitoterápicos para a recuperação e manutenção da saúde representa um mercado farmacêutico bastante promissor. Em 2010 o mercado mundial de fitoterápicos movimentou cerca de US\$ 44 bilhões, e apesar de não existirem dados oficiais sobre o tamanho desse mercado no Brasil, as estimativas variam entre US\$ 350 milhões e US\$ 550 milhões, segundo a Associação Brasileira de Empresas do Setor Fitoterápico (ABIFISA). ${ }^{1,2}$

A produção de fitoterápicos envolve um processo interdisciplinar, multidisciplinar e interinstitucional e abrange diversas etapas. Uma destas consiste no emprego de métodos analíticos para quantificação e controle dos marcadores químicos ou farmacológicos, presentes tanto na matéria-prima vegetal quanto no produto intermediário e final. Esses métodos compreendem requisitos indispensáveis ao registro desses produtos no Brasil, tendo em vista que permitem a avaliação de sua qualidade, eficácia terapêutica e segurança. ${ }^{3,4}$

Entre as inúmeras espécies vegetais de interesse medicinal e que despertam o interesse para produção de fitoterápicos, encontra-se a Bauhinia forficata Link (pata-de-vaca), que se destaca pela sua relevância terapêutica no tratamento do diabetes mellitus e por se encontrar na Relação Nacional de Plantas Medicinais de Interesse ao Sistema Único de Saúde (RENISUS), divulgada em 2009 pelo Ministério da Saúde.$^{5,6}$ Essa espécie, pertencente ao gênero Bauhinia e família Fabaceae, é considerada uma planta nativa da América do Sul, encontrada no Brasil, predominantemente, entre as regiões do Rio de Janeiro e Rio Grande do Sul. ${ }^{7,8}$

Os estudos fitoquímicos e farmacológicos realizados com $B$. forficata sugerem os glicosídeos flavonoídicos canferólicos e quercetínicos como seus principais constituintes e prováveis responsáveis pela sua propriedade antidiabética, sendo o canferol 3,7-di-O- $\alpha-L-$ rhamnopiranosídeo (canferitrina) considerado o marcador analítico das folhas. ${ }^{7,9}$ Apesar da potencialidade farmacológica e do grande interesse pela comunidade farmacêutica, a espécie referida não consta em monografias oficiais, fazendo-se necessário o desenvolvimento e a validação de métodos analíticos para quantificação de seus compostos flavonoídicos e padronização de extratos. ${ }^{10}$

Diversas técnicas são empregadas para o doseamento de flavonoi-

*e-mail: phtech@uol.com.br des em materiais vegetais, uma delas, a espectrofotometria de absorção na região do ultravioleta-visível (UV-Vis) alcança grande destaque devido a sua simplicidade, rapidez, baixo custo de execução e ampla disponibilidade nos laboratórios de controle de qualidade. ${ }^{11,12}$ Embora extremamente utilizada, a pouca seletividade tem sido um dos maiores desafios dessa técnica para análise de matrizes complexas como os extratos vegetais, pois a espectrofotometria direta pode ocasionar sobreposição das bandas, impedindo a absorção do componente de interesse. ${ }^{13,14}$ Decorrente disso, para obtenção dos espectros dos flavonoides sem a interferência de outros compostos fenólicos, comumente se emprega o método colorimétrico com cloreto de alumínio $\left(\mathrm{AlCl}_{3}\right)$ para tratamento das amostras a serem analisadas. Isto porque o cátion $\mathrm{Al}^{3+}$ forma complexos estáveis com as hidroxilas livres dos flavonoides, ocasionando extensão do sistema conjugado e, consequentemente, um desvio batocrômico, ou seja, um deslocamento dos seus máximos de absorção para regiões de maior comprimento de onda. ${ }^{15-17}$

Entretanto, antes de sua adoção oficial, os métodos ou procedimentos analíticos precisam ser submetidos a uma série de experimentos capazes de avaliar sua capacidade em detectar e quantificar um determinado analito. ${ }^{12,18}$ De acordo com a Resolução Específica (RE) nº 899/03 da Agência Nacional de Vigilância Sanitária (ANVISA), essa validação deve ser realizada através da análise de uma série de parâmetros, quais sejam: especificidade, linearidade, limite de detecção (LD), limite de quantificação (LQ), precisão, exatidão e robustez, sendo o principal objetivo atestar a eficiência dos métodos na rotina laboratorial. ${ }^{19}$

Portanto, impulsionados pelas necessidades normativas no que se refere à regularização de fitoterápicos e diante do amplo interesse popular e institucional acerca da B. forficata, esse estudo descreve a avaliação dos procedimentos para determinação espectrofotométrica de flavonoides totais descritos na literatura, com e sem hidrólise ácida, aplicados às folhas dessa espécie, de forma que os mesmos atendam às exigências sanitárias em vigor. ${ }^{19}$

\section{PARTE EXPERIMENTAL}

\section{Material vegetal}

As folhas de B. forficata foram coletadas em dezembro/2009 na Vila Gianetti, Viçosa - MG, sendo a identificação realizada pelo Prof. 
J. M. Fernandes e a exsicata depositada no Herbário VIC, do Departamento de Biologia Vegetal, na Universidade Federal de Viçosa, sob o $n^{\circ}$ 32513. A identidade da amostra foi confirmada no Jardim Botânico do Rio de Janeiro - RJ, pela especialista em identificação de espécies do gênero Bauhinia, A. Vaz. Na preparação da matéria-prima vegetal, as folhas foram submetidas à secagem em estufa a uma temperatura de $45^{\circ} \mathrm{C}$, durante $72 \mathrm{~h}$ e, em seguida, pulverizadas em liquidificador industrial (Bermar ${ }^{\circledR}$ BM30).

\section{Reagentes e vidrarias}

Todos os reagentes utilizados foram de grau analítico: cloreto de alumínio $\left(\operatorname{Vetec}^{\circledR}\right)$, acetona, ácido clorídrico, acetato de etila, metanol, ácido acético, ácido fosfórico, etanol (Dinâmica ${ }^{\circledR} ;$ FMaia $^{\circledR}$ ), acetona $\left(\right.$ Cinética $\left.^{\circledR}\right)$, metenamina, quercetina e rutina $\left(\right.$ Merck $\left.^{\circledR}\right)$. Utilizaram-se vidrarias volumétricas calibradas, com certificado de calibração por lote do fabricante Satelit ${ }^{\circledR}$.

\section{Equipamentos}

Os equipamentos utilizados foram balança analítica Bioprecisa ${ }^{\circledR}$, modelo FA2104N, banho-maria Nova ética ${ }^{\circledR}$ e espectrofotômetro UV-Vis mini- 1240 Shimadzu ${ }^{\circledR}$.

\section{Teor de flavonoides totais por espectrofotometria}

Procedimento por diluição direta (PDD) - sem hidrólise

\section{Preparação da solução extrativa}

A solução extrativa foi obtida através de extração sob refluxo com solução hidroalcoólica $(40 \%, v / v)$, baseando-se no método desenvolvido e validado previamente por Petry e colaboradores. ${ }^{20} \mathrm{~A}$ extração foi realizada em balão de fundo redondo contendo $1,0 \mathrm{~g}$ do material vegetal e $30 \mathrm{~mL}$ de solução hidroalcoólica, durante $30 \mathrm{~min}$. $\mathrm{O}$ extrato foi resfriado à temperatura ambiente $\left(25^{\circ} \mathrm{C}\right)$ e filtrado em algodão, sendo o resíduo (algodão e material vegetal) re-extraído por mais duas vezes, durante $10 \mathrm{~min}$. As frações filtradas foram reunidas em balão volumétrico e o volume ajustado para $100,0 \mathrm{~mL}$ com a solução hidroalcoólica $(40 \%, \mathrm{v} / \mathrm{v})$.

\section{Preparação da amostra para leitura}

Alíquotas da solução extrativa foram transferidas para balões volumétricos de $25 \mathrm{~mL}$, onde se adicionaram $2,0 \mathrm{~mL}$ de solução etanólica de $\mathrm{AlCl}_{3}(5 \%, \mathrm{~m} / \mathrm{v})$. Ajustou-se o volume, em seguida, com a solução hidroalcoólica $(40 \%, v / v)$. Após a adição da solução de $\mathrm{AlCl}_{3}(5 \%, \mathrm{~m} / \mathrm{v})$, a absorvância foi medida em espectrofotômetro, utilizando-se como branco uma solução de compensação, preparada do mesmo modo que a amostra, mas sem adição da solução etanólica de $\mathrm{AlCl}_{3}(5 \%, \mathrm{~m} / \mathrm{v})$.

\section{Determinação da concentração da amostra e comprimento de onda para leitura}

Uma série de amostras, diluídas a partir de uma solução extrativa $(40 \%, \mathrm{v} / \mathrm{v})$, foram submetidas à varredura em espectrofotômetro na faixa de 230 a $500 \mathrm{~nm}$, após $30 \mathrm{~min}$ da adição de solução etanólica de $\mathrm{AlCl}_{3}(5 \%, \mathrm{~m} / \mathrm{v})$, para identificação da diluição e do comprimento de onda que apresentassem o valor de absorbância mais adequado para o método.

\section{Determinação do tempo para leitura}

Foi realizada através da leitura de amostras da solução extrativa em comprimento de onda de $408 \mathrm{~nm}$, em intervalos de 5, 15, 30, 45 e 60 min após adição da solução etanólica de $\mathrm{AlCl}_{3}$.
Procedimento por hidrólise ácida (PHA)

\section{Preparação da solução extrativa}

A solução extrativa foi obtida através de extração sob refluxo de 1,0 g da droga vegetal em solução ácida de acetona na presença de metenamina 0,5\%, conforme preconizado pela Farmacopeia Brasileira para Calendula officinalis L. e modificado por Pinheiro e colaboradores. $^{21}$

\section{Preparação da amostra para leitura}

Alíquotas da solução extrativa foram transferidas para balões volumétricos de $25 \mathrm{~mL}$, onde se adicionou $1,0 \mathrm{~mL}$ de solução metanólica de $\mathrm{AlCl}_{3}(5 \%, \mathrm{~m} / \mathrm{v})$. Ajustou-se o volume, em seguida, com solução metanólica de ácido acético $(2,5 \%$, v/v). Após a adição da solução de $\mathrm{AlCl}_{3}(5 \%, \mathrm{~m} / \mathrm{v})$, a absorvância foi medida em espectrofotômetro, utilizando-se como branco uma solução de compensação, preparada do mesmo modo que a amostra, mas sem adição da solução metanólica de $\mathrm{AlCl}_{3}(5 \%, \mathrm{~m} / \mathrm{v})$.

\section{Determinação da concentração da amostra e comprimento de onda para leitura}

Uma série de amostras, diluídas a partir da solução extrativa, foram submetidas à varredura em espectrofotômetro na faixa de 230 a $500 \mathrm{~nm}$, após 30 min da adição de solução metanólica de $\mathrm{AlCl}_{3}(5 \%$, $\mathrm{m} / \mathrm{v}$ ), para identificação da diluição e do comprimento de onda que apresentassem o valor de absorbância mais adequado para o método.

\section{Determinação do tempo para leitura}

Foi realizada através da leitura de amostras da solução extrativa em comprimento de onda de $408 \mathrm{~nm}$, em intervalos de 5, 15, 30, 45 e 60 min após adição da solução metanólica de $\mathrm{AlCl}_{3}$.

\section{Cálculo para determinação do teor de flavonoides totais (TFT)}

O teor de flavonoides totais, expresso em porcentagem (p/p) de flavonoides totais calculados como quercetina, foi calculado para ambos os procedimentos de acordo com a Equação 1:

$$
T F T=\frac{A \times F D}{m \times E_{1 \mathrm{~cm}}^{1 \%}}
$$

onde: $\mathrm{TFT}=$ teor de flavonoides totais; $\mathrm{A}=$ absorvância determinada; $\mathrm{FD}=$ fator de diluição; $\mathrm{m}=$ massa de droga seca $(\mathrm{g}) ; E_{1 c m}^{1 \%}=$ absorção específica do complexo quercetina - cloreto de alumínio.

Posteriormente, as concentrações foram convertidas para $\mu \mathrm{g}$ de quercetina por $\mathrm{mL}$ de extrato.

\section{Avaliação dos procedimentos analíticos}

Avaliação da razão droga vegetal:solvente

Para ambos os procedimentos estudados foram preparadas soluções extrativas a partir de diferentes quantidades de droga vegetal: 0,$5 ; 0,75 ; 1,0 ; 1,25$ e $1,5 \mathrm{~g}$. Desta forma, foi avaliada a influência da razão de droga vegetal:líquido extrator (p:v) sobre a resposta do procedimento.

\section{Avaliação da influência da diluição do analito e da concentração de reagente}

A avaliação destes parâmetros foi conduzida através de um planejamento fatorial $3^{2}$ (2 variáveis em 3 níveis de variação) aumentado de 2 pontos centrais para avaliação do efeito da concentração das amostras e concentração das soluções de $\mathrm{AlCl}_{3}$ (variáveis independentes) sobre o comportamento espectrofotométrico dos flavonoides 
(variável dependente). A Tabela 1 apresenta os valores utilizados em cada nível estudado. Os níveis de concentração das amostras foram selecionados nos estudos preliminares, de acordo com o item Determinação da concentração da amostra e comprimento de onda, enquanto os níveis de concentração da solução de $\mathrm{AlCl}_{3}$ foram baseados no estudo realizado Petry e colaboradores. ${ }^{22}$ Todas as análises foram realizadas em triplicata e de modo aleatório. A análise estatística foi realizada com o auxílio do software Statistica ${ }^{\circledR}, 6.0$ (StatSoft, USA), através do qual foram geradas superfícies de resposta.

\section{Validação dos procedimentos analíticos}

Os procedimentos descritos foram avaliados quanto à especificidade, linearidade, limites de detecção e de quantificação, precisão (repetitividade e precisão intermediária), exatidão e robustez, de acordo com as normas estabelecidas. ${ }^{19}$ Todas as análises foram realizadas em triplicata e a confiabilidade dos parâmetros foi verificada pelo coeficiente de variação percentual $(\mathrm{CV} \%)$ ou desvio padrão relativo (DPR\%), não se admitindo valores superiores a 5\%. Adicionalmente, os resultados foram tratados estatisticamente por análise de variância (ANOVA) One-Way ou Two-Way, quando aplicável.

\section{Especificidade}

A especificidade das técnicas foi demonstrada através da sobreposição dos espectros de padrões e das amostras do extrato com e sem adição da solução de $\mathrm{AlCl}_{3}(2,5 \%$, m/v), obtidos na faixa de 230 a 500 $\mathrm{nm}$. Quercetina foi empregada para avaliação do procedimento PDD, enquanto que a rutina foi adotada para avaliação do procedimento PHA. No segundo caso, a rutina foi previamente tratada de acordo com o procedimento de hidrólise aplicado à droga vegetal. A eleição das substâncias dos padrões quercetina e rutina foi fundamentada nos relatos da presença dos mesmos na espécie estudada e na similaridade da estrutura com os glicosídeos canferólicos.

\section{Linearidade}

A linearidade da resposta espectrofotométrica para os procedimentos em estudo foi verificada a partir da análise de três curvas autênticas, construídas com amostras da solução extrativa, em cinco níveis de concentração, nas faixas de 0,8 a 4,0 e de 0,3 a 0,9 mg/ $\mathrm{mL}$ para PDD e PHA, respectivamente. As curvas foram construídas empregando-se os valores médios das absorvâncias (PDD e PHA) em função da concentração. Os resultados obtidos foram tratados estatisticamente através do cálculo de regressão linear pelo método dos mínimos quadrados, a fim de definir o coeficiente de determinação $\left(R^{2}\right)$, adotando $R^{2}>0,99$ como valor mínimo para aceitação.

\section{Limites de detecção e quantificação}

Os limites de detecção (LD) e quantificação (LQ) foram estimados $(\mathrm{em} \mu \mathrm{g} / \mathrm{mL})$ de acordo com as equações $\mathrm{LD}=\mathrm{DP}_{\mathrm{a}} \times 3 / \mathrm{IC}$ e LQ $=$ $\mathrm{DP}_{\mathrm{a}} \times 10 / \mathrm{IC}$, onde $\mathrm{DP}_{\mathrm{a}}$ é o desvio padrão do intercepto com o eixo $\mathrm{Y}$, obtido das três curvas de linearidade e IC é a média dos coeficientes angulares (inclinação da reta) das respectivas curvas.

\section{Precisão}

A precisão foi avaliada pela repetitividade (precisão intracorrida), na qual foram examinadas, em um único dia, 6 determinações individuais para amostras a $100 \%$ da concentração teste, obtidas a partir de soluções extrativas diferentes, e pela precisão intermediária (precisão intercorridas), a qual foi determinada por dois analistas em dois dias consecutivos, para amostras também na concentração de $100 \%$.

\section{Exatidão}

A exatidão foi avaliada por ensaios de recuperação, através da adição de quantidades conhecidas de solução extrativa diluída a amostras a $100 \%$ da concentração teste. Os valores de recuperação, expressos em porcentagem, foram determinados através da razão entre as concentrações médias determinadas experimentalmente com as concentrações teóricas correspondentes. Para a PDD, amostras a $2,8 \mathrm{mg} / \mathrm{mL}$ de material vegetal foram acrescidas de quantidades crescentes $(1,0 ; 2,0$ e 3,0 mL) de uma solução contaminante a 3,6 mg/ $\mathrm{mL}$ de material vegetal, resultando em três soluções $(2,944 ; 3,088 \mathrm{e}$ $3,232 \mathrm{mg} / \mathrm{mL}$ ), com três réplicas cada. Do mesmo modo, para a PHA, amostras $(0,64 \mathrm{mg} / \mathrm{mL})$ foram acrescidas de quantidades crescentes de uma solução extrativa contaminante $(1,12 \mathrm{mg} / \mathrm{mL})$, resultando em três soluções $(0,6848 ; 0,7296$ e $0,7744 \mathrm{mg} / \mathrm{mL})$, com três réplicas cada.

\section{Robustez}

O ensaio para determinação da robustez dos procedimentos sobre a resposta espectrofotométrica foi realizado a partir da variação dos seguintes parâmetros: influência da luminosidade (presença e ausência de luz), estabilidade da solução extrativa ( 0,3 e 6 h), fabricante do solvente [álcool etílico absoluto (Dinâmica ${ }^{\circledR}$ e FMaia ${ }^{\circledR}$ ) para a PDD e acetona (Dinâmica ${ }^{\circledR}$ e Cinética ${ }^{\circledR}$ ) para a PHA].

\section{RESULTADOS E DISCUSSÃO}

\section{Avaliação dos procedimentos analíticos}

\section{Determinação da concentração da amostra e comprimento de onda para leitura}

Os comprimentos de onda com absorvância máxima observados nos espectros de absorção das amostras obtidas pelo PDD e PHA, após a reação de complexação com $\mathrm{AlCl}_{3}$, foram de 408 e $421 \mathrm{~nm}$, respectivamente. Já as diluições da solução extrativa que apresentaram o valor de absorbância mais adequado para os procedimentos foram de 7/25 e 4/25 v/v, correspondendo às concentrações de 2,8 e $0,64 \mathrm{mg} / \mathrm{mL}$.

\section{Determinação do tempo de leitura}

O tempo de reação necessário para formação do complexo flavonóide- $\mathrm{Al}^{3+}$ exerce função importante na resposta de métodos espectrofotométricos e depende de fatores tais como padrão de substituição da aglicona e da razão aglicona- $\mathrm{Al}^{3+}$.22 Também merece atenção o fato de que em misturas ou em extratos, o perfil de formação destes complexos é diferente do observado para substâncias puras. Portanto, a avaliação e o estabelecimento do tempo necessário para que haja formação máxima do complexo, assim como a estabilidade depois de formado, é uma das etapas preliminares para que este erro sistemático possa ser minimizado. ${ }^{20}$ Neste estudo, comportamentos distintos foram observados para as amostras estudas. Embora ambas as amostras tenham apresentado aumento na absorvância até 30 min de reação após a adição de solução de $\mathrm{AlCl}_{3}$, o emprego do procedimento de diluição direta (PDD) propiciou comportamento estável até o final do ensaio. Por outro lado, a amostra obtida após hidrólise ácida (PHA) apresentou redução na absorvância lida após 30 min, com redução importante da resposta até o fim do experimento (Figura 1). Embora haja reversão dos complexos formados na segunda amostra, os dados obtidos permitem estabelecer o tempo de leitura de 30 min, após adição da solução de $\mathrm{AlCl}_{3}$, como o mais apropriado para realização da leitura.

\section{Avaliação da proporção droga vegetal:solvente}

A influência da proporção de droga vegetal sobre a resposta do método para ambos os procedimentos analíticos foi avaliada na faixa de 0,5 a 1,5 g. Os dados revelaram comportamento linear para os teores de flavonoides totais (TFT) calculados para amostras na 

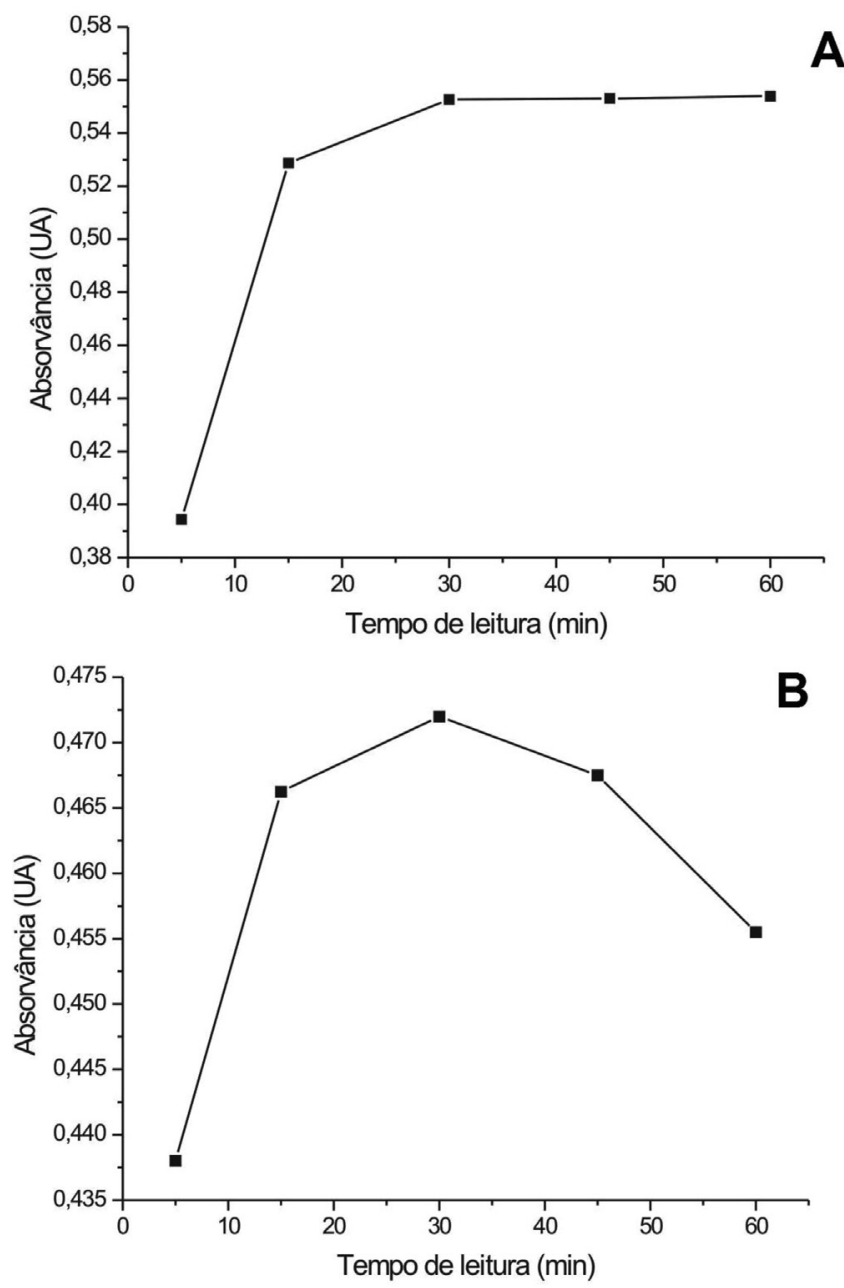

Figura 1. Cinética de complexação dos flavonoides com $\mathrm{AlCl}_{3}$. (A) PDD $e$ (B) PHA

região de 0,5 a 1,0 g. Para amostras de 1,25 g ou superior, a resposta do método apresentou decréscimo significativo para o TFT, o qual pode ser atribuído à saturação do meio de extração ou limitação do meio reacional. Por outro lado, amostras menores que $0,5 \mathrm{~g}$ de droga vegetal levaram a leituras inferiores a 0,2 U.A., as quais estão em desacordo com a lei de Lambert-Beer. ${ }^{23}$ Considerando as limitações observadas, os TFTs calculados para amostras de droga vegetal na região linear foram de 45,60 $\pm 1,68 \mu \mathrm{g} / \mathrm{mL}(3,69 \%)$ e 168,36 $\pm 0,81$ $\mu \mathrm{g} / \mathrm{mL}(0,48 \%)$, para PDD e PHA, respectivamente.

\section{Avaliação da influência da diluição do analito e da concentração de reagente}

Como discutido anteriormente, a formação do complexo flavonoide- $\mathrm{Al}^{3+}$ apresenta dependência importante das diferentes classes de flavonoides e esse efeito é bastante específico e intrínseco. Ademais, o comportamento observado para o flavonoide isolado sofre modificação importante quando presente em mistura ou na solução extrativa, de maneira que para cada droga vegetal é primordial investigar o comportamento antes da adoção de procedimentos desta natureza. ${ }^{24}$ Nesse sentido, as influências das concentrações de $\mathrm{AlCl}_{3}$ e da amostra, sobre a resposta do método para cada procedimento proposto, foram investigadas através de planejamento fatorial do tipo $3^{2}$, e adicionados de 2 pontos centrais A matriz completa do planejamento experimental para avaliação de ambos os procedimentos (PDD e PHA) está apresentada na Tabela 1, juntamente com os resultados experimentas encontrados.

Os dados foram empregados para gerar superfícies de respostas (Figura 2), as quais permitiram observar o comportamento dos fatores (variáveis independentes) e suas interações sobre a resposta espectrofotométrica (variável dependente) para cada procedimento analítico (PDD ou PHA). ${ }^{24}$

A análise das superfícies de resposta (Figura 2) revelou que, independente do procedimento de preparação de amostra, as respostas do método (absorvância) não foram sensíveis à variação nas concentrações de $\mathrm{AlCl}_{3}$. Considerando a concentração de droga vegetal, a resposta do método apresentou relação linear e não foi observada qualquer interação entre os fatores estudados. Assim, os resultados encontrados permitiram a escolha da solução de $\mathrm{AlCl}_{3}(2,5 \%, \mathrm{~m} / \mathrm{v})$ como reagente para qualquer dos procedimentos adotados.

\section{Validação dos procedimentos analíticos}

\section{Especificidade}

Na Figura 3 estão apresentados os espectros de varredura obtidos para as soluções de referência de quercetina e hidrolisado da rutina após adição de $\mathrm{AlCl}_{3}$, em comparação com os espectros das soluções extrativas de $B$. forficata antes e após a adição de $\mathrm{AlCl}_{3}$ e obtidas de acordo com ambos os procedimento PDD e PHA. A avaliação dos espectros permitiu observar que há similaridade importante entre os espectros das soluções empregadas como referência e as respectivas soluções extrativas. Considerando o procedimento PDD (Figura 3A),

Tabela 1. Matriz do planejamento experimental

\begin{tabular}{lccccccc}
\hline \multirow{2}{*}{ Exp. } & \multicolumn{2}{c}{ Variáveis codificadas } & \multicolumn{3}{c}{ Variáveis naturais } & \multicolumn{2}{c}{ Abs (U.A.) } \\
\cline { 2 - 7 } & $\mathrm{AlCl}_{3}$ & Amostra & $\mathrm{AlCl}_{3}(\% \mathrm{~m} / \mathrm{v})$ & $\mathrm{PDD}(\mathrm{mg} / \mathrm{mL})$ & $\mathrm{PHA}(\mathrm{mg} / \mathrm{mL})$ & PDD (média $\pm \mathrm{dp})$ & PHA (média $\pm \mathrm{dp})$ \\
\hline 1 & -1 & -1 & 2,5 & 1,6 & 0,32 & $0,357 \pm 0,01$ & $0,231 \pm 0,01$ \\
2 & -1 & 0 & 2,5 & 2,8 & 0,64 & $0,550 \pm 0,01$ & $0,453 \pm 0,01$ \\
3 & -1 & 1 & 2,5 & 4,0 & 0,96 & $0,738 \pm 0,01$ & $0,668 \pm 0,01$ \\
4 & 0 & -1 & 5,0 & 1,6 & 0,32 & $0,351 \pm 0,01$ & $0,240 \pm 0,01$ \\
5 & 0 & 0 & 5,0 & 2,8 & 0,64 & $0,565 \pm 0,01$ & $0,469 \pm 0,01$ \\
6 & 0 & 1 & 5,0 & 4,0 & 0,96 & $0,755 \pm 0,01$ & $0,690 \pm 0,01$ \\
7 & 1 & -1 & 7,5 & 1,6 & 0,32 & $0,343 \pm 0,01$ & $0,243 \pm 0,01$ \\
8 & 1 & 0 & 7,5 & 2,8 & 0,64 & $0,539 \pm 0,01$ & $0,473 \pm 0,01$ \\
9 & 1 & 1 & 7,5 & 4,0 & 0,96 & $0,755 \pm 0,01$ & $0,685 \pm 0,01$ \\
10 & 0 & 0 & 5,0 & 2,8 & 0,64 & $0,548 \pm 0,01$ & $0,467 \pm 0,01$ \\
11 & 0 & 0 & 5,0 & 2,8 & 0,64 & $0,546 \pm 0,01$ & $0,464 \pm 0,01$ \\
\hline
\end{tabular}



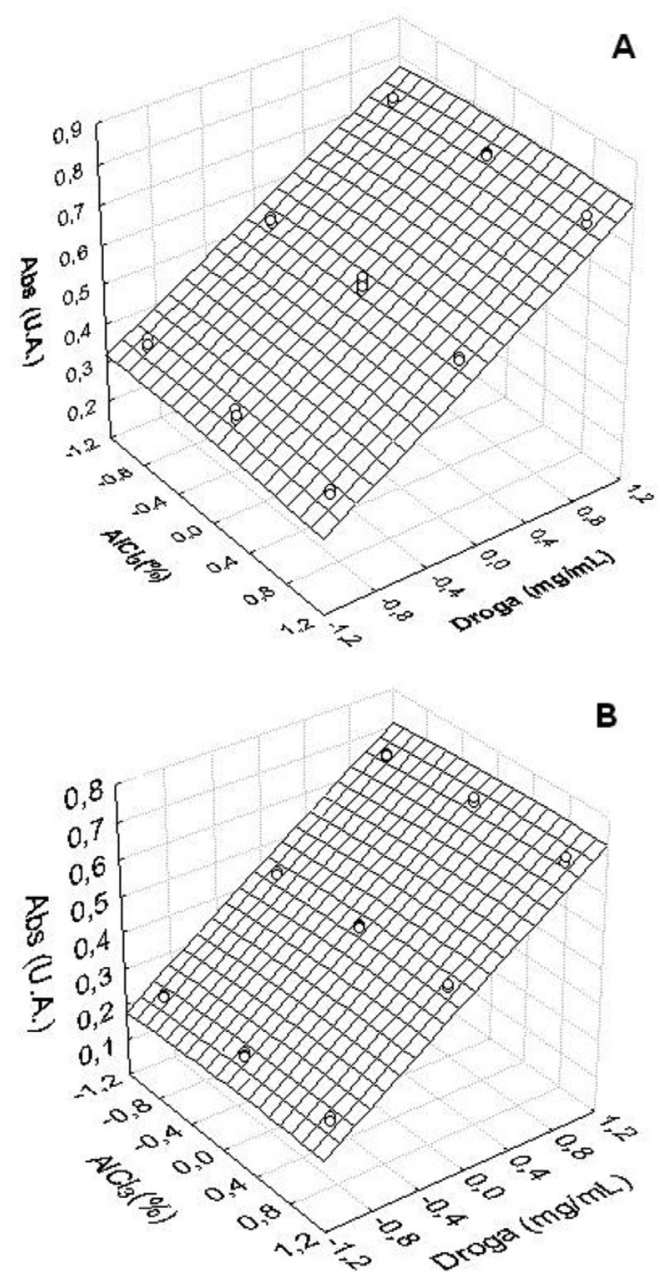

Figura 2. Superfícies de respostas geradas a partir das respostas do planejamento fatorial $3^{2}$ para PDD (A) e PHA (B). [Abs] = Absorvância

após a adição da $\mathrm{AlCl}_{3}$ o comprimento de onda onde ocorre o máximo de absorção foi de $408 \mathrm{~nm}$. Por outro lado, para o procedimento PHA (Figura 3 B), o máximo observado após adição de $\mathrm{AlCl}_{3}$ ocorreu em $421 \mathrm{~nm}$. Os dados apresentados confirmam que os comprimentos de onda selecionados anteriormente representam satisfatoriamente os marcadores eleitos para a espécie.

Linearidade, limites de detecção $(L D)$ e quantificação $(L Q)$

Para avaliar a linearidade da resposta analítica para ambos os procedimentos, foi executada regressão linear para as curvas obtidas a partir de cinco concentrações nas regiões de 0,8 a 4,0 e 0,3 a 0,9 $\mathrm{mg} / \mathrm{mL}$, para os procedimentos PDD e PHA, respectivamente. Os dados da regressão obtidos pelo método dos mínimos quadrados apresentaram valores de coeficientes de determinação $\left(R^{2}\right)$ de 0,998 e 0,999 para o PDD e PHA, respectivamente, denotando que mais que $99 \%$ (99,8 e 99,9\%, respectivamente), da variabilidade experimental é explicada satisfatoriamente pela equação proposta, comprovando a relação linear entre o aumento da concentração do analito e a resposta espectrofotométrica. Os dados permitiram afirmar que o desempenho dos procedimentos atende os requisitos preconizados para análise desse parâmetro.

Considerando os limites de detecção (LD) e de quantificação (LQ), os resultados encontrados foram 0,130 e $0,435 \mathrm{mg} / \mathrm{mL}$ e 0,028 e $0,091 \mathrm{mg} / \mathrm{mL}$ para PDD e PHA, respectivamente. Com esses resultados, verificou-se que os procedimentos proporcionam respostas espectrofotométricas com sensibilidade para detecção e
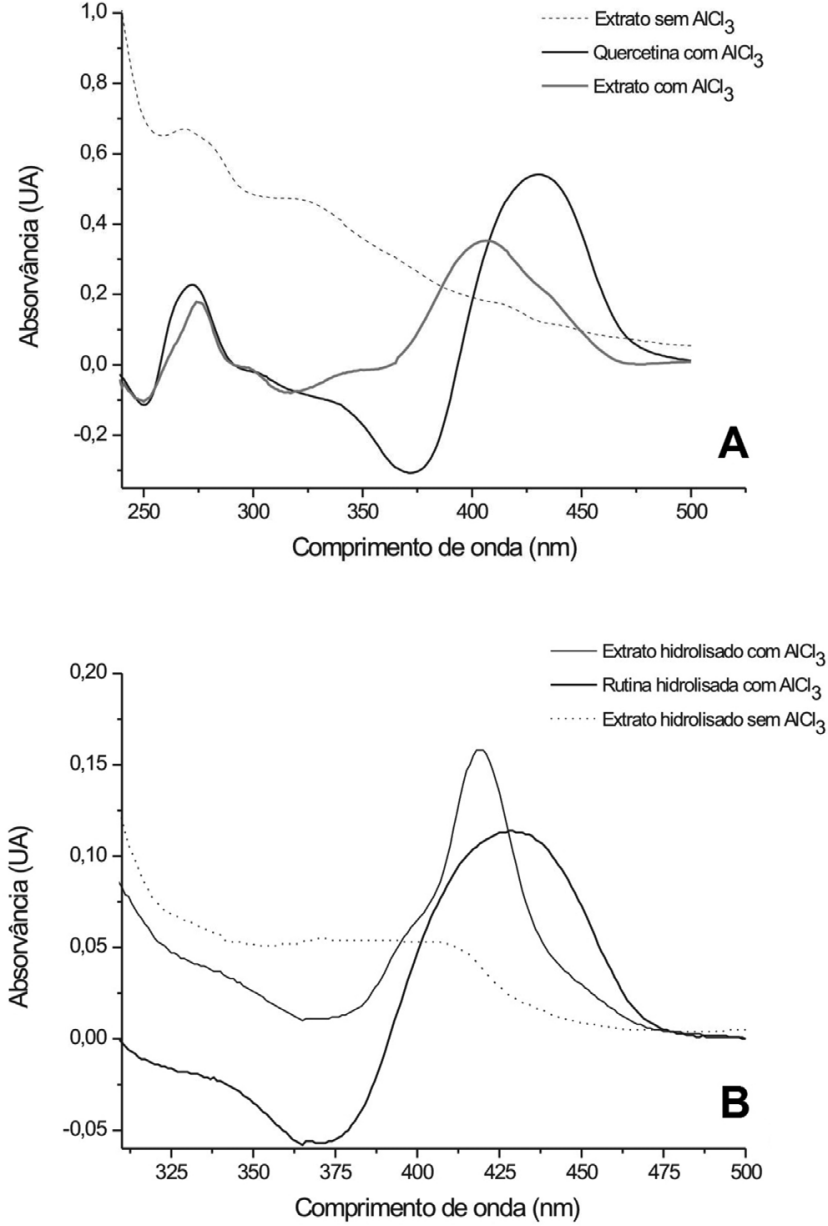

Figura 3. Espectros de varredura obtidos na análise da especificidade. (A) $P D D$ e (B) PHA

quantificação dos flavonoides totais nos extratos de B. forficata com a confiabilidade desejada.

Precisão, exatidão e robustez

Os procedimentos propiciaram respostas espectrofotométricas precisas nos dois níveis analisados: repetitividade e precisão intermediária. Quanto ao parâmetro repetitividade para PDD e PHA, os resultados apresentaram, respectivamente, respostas médias em flavonoides totais de 41,53 e $164,99 \mu \mathrm{g} / \mathrm{mL}$ e CVs de 0,13 e $0,85 \%$ (valores bem abaixo do valor máximo preconizado e igual 5\%). Quanto ao parâmetro precisão intermediária, foi demonstrado, através do tratamento estatístico por ANOVA de duas entradas (two-way ANOVA), que os procedimentos são precisos para as análises realizadas por analistas diferentes em um mesmo dia e em dias diferentes $(p<0,05)$.

A exatidão dos procedimentos foi estimada a partir do ensaio de recuperação. Desta forma, a interferência da matriz sobre a resposta do método foi avaliada sob dois aspectos: a capacidade de extração dos analitos e a repetibilidade das respostas. De acordo com os dados obtidos, não houve interferência significativa dos procedimentos sobre a recuperação de flavonoides totais, os quais apresentaram taxas de recuperação de $100,55 \pm 1,71 \%$ para PDD e $100,46 \pm 1,26 \%$ para PHA. Adicionalmente, os procedimentos apresentaram respostas bastante exatas em todos os níveis testados, confirmados através dos coeficientes de variação de $1,70 \%$ para PDD e 1,25\% para PHA.

A robustez dos procedimentos foi testada preliminarmente através da avaliação da fotoestabilidade dos complexos (exposição à luz do dia por até $6 \mathrm{~h}$ ) e do fabricante do etanol. Os dados mostraram que ambos os procedimentos (PDD e PHA) se mostraram robustos quanto 
aos parâmetros analisados, pois todos os $\mathrm{F}$ calculados foram inferiores aos $\mathrm{F}$ tabelados, através de ANOVA de uma entrada (one-way ANOVA).

\section{CONCLUSÃO}

Os procedimentos analíticos para determinação espectrofotométrica de flavonoides totais nas folhas de B. forficata, avaliados neste trabalho, apresentaram desempenho dentro das especificações sanitárias preconizadas para métodos bioanalíticos para os parâmetros de linearidade, especificidade, sensibilidade, exatidão e recuperação. Desta forma, os procedimentos puderam ser considerados validados para o fim que se propõem. Enquanto o procedimento por diluição direta apresentou maior simplicidade em virtude do menor número de passos experimentais, o procedimento por hidrólise ácida apresentou maior sensibilidade em virtude do procedimento de preparação da amostra. Por fim, foi possível concluir que, independente das metodologias, ambos os procedimentos avaliados neste estudo estão apropriados ao emprego como ferramenta de controle de qualidade para a droga vegetal e produtos derivados das folhas de $B$. forficata.

\section{MATERIAL SUPLEMENTAR}

As tabelas com os resultados da análise de precisão intermediária, da exatidão e robustez encontram-se disponíveis em http:// quimicanova.sbq.org.br, na forma de arquivo PDF, com acesso livre.

\section{AGRADECIMENTOS}

Ao suporte financeiro do CNPq, CAPES e FACEPE, além do auxílio do Prof. MSc. C. P. Ferreira para a obtenção do material vegetal.

\section{REFERÊNCIAS}

1. Araújo, A. A. S.; Mercuri, A. L. P.; Seixas, S. R. S.; Storpirtis, S.; Matos, J. R.; Rev. Bras. Cienc. Farm. 2006, 42, 269.

2. http://www.abifisa.org.br/noticias_ver.asp?news=3594, acessada em Novembro 2011.

3. Toledo, A. C. O.; Hirata, L. L.; Buffon, M. C. M.; Miguel, M. D.; Miguel, O. G.; Rev. Lecta 2003, $21,7$.

4. Brasil; Resolução RDC n ${ }^{\circ} 14$ de 31/03/10; Diário Oficial da União, Brasília, DF, 05/04/10, seção 1 - Agência Nacional de Vigilância Sanitária (ANVISA) dispõe sobre o registro de medicamentos fitoterápicos.
5. Silva, K. L.; Cechinel Filho, V.; Quim. Nova 2002, 25, 449.

6. http://portal.saude.gov.br/portal/arquivos/pdf/RENISUS.pdf, acessada em Novembro 2011

7. Pizzollatti, M. G.; Cunha Jr., A.; Szpoganicz, B.; Souza. E.; Quim. Nova 2003, 26, 466.

8. Vaz, A. M. S. F.; Tozzi, A. M. G. A.; Rev. Bras. Bot. 2005, $28,477$.

9. Sousa, E.; Zanatta L.; Seifriz, I.; Creczynski-Pasa, T. B.; Pizzolatti, M. G.; Szpoganicz, B.; Silva, F. R. M. B.; J. Nat. Prod. 2004, 67, 829.

10. Lusa, M. G.; Bona, C.; Acta Bot. Bras. 2009, 23, 196.

11. Komarova, N. I.; Rogachev, A. D.; Chernyak, E. I.; Morozov, S. V.; Fomenko, V. V.; Salakhutdinov, N. F.; Chem. Nat. Compd. 2009, 45, 27.

12. Alves, L. D. S.; Rolim, L. A.; Fontes, D. A. F.; Rolim-Neto, P. J.; La Roca, M. F.; Soares Sobrinho, J. L.; Quim. Nova 2010, 33, 1967.

13. Perkampus, H. H.; UV-VIS spectroscopy and its applications, SpringerVerlag: Berlin, 1992.

14. Rocha, F. R. P.; Teixeira, L. S. G.; Quim. Nova 2004, 27, 807.

15. Marcucci, M. C.; Woisky, R. G.; Salatino, A.; Mensagem Doce 1998, 46, 3.

16. Souza, R. F. V.; Giovani, W. R. F.; Spectrochim Acta, Part A 2005, 61, 1985.

17. Buriol, L.; Finger, D.; Schmidt, E. M.; dos Santos, J. M. T.; da Rosa, M. R.; Quináia, S. P.; Torres, Y. N.; Santa, H. S. D.; Pessoa, C.; de Moraes, M. O.; Costa-Lotufo, L. V.; Ferreira, P. M. P.; Sawaya, A. C. H. F.; Eberlin, M. N.; Quim. Nova 2009, 32, 296.

18. Santana, A. K. M.; Nunes, L. C. C.; Medeiros, F. P. M.; Silva, M. J.; Lavra, Z. M. M.; Rolim-Neto, P. J.; Rev. Ciênc. Farm. Básica Apl. 2007, 28, 177.

19. Brasil; Resolução RE no899 de 29/5/2003; Diário Oficial da União, Brasília, DF, 02/06/2003, seção 1 - Agência Nacional de Vigilância Sanitária (ANVISA) aprova Guia para validação de métodos analíticos e bioanalíticos.

20. Petry, R. D.; De Souza, K. C. B.; Bassani, V. L.; Petrovick, P. R.; González Ortega, G.; Rev. Bras. Farm. 1998, 79, 7.

21. Pinheiro, T. S. D. B.; Johansson, L. A. P.; Pizzolatti, M.G.; Biavatti, M. W.; J Pharmaceut. Biomed. Anal. 2006, 41, 431.

22. Petry, R. D.; Gonzáles Ortega, G.; Silva, W. B.; Die Pharmazie 2001, $56,465$.

23. Soares, L. A. L.; Bassani, V. L.; González Ortega, G.; Petrovick, P. R.; Acta Farm. Bonaerense 2003, 22, 203.

24. Mothé, C. G.; Correia, D. Z.; Rev. Analytica 2002, 2, 59. 


\section{AVALIAÇÃO DE PROCEDIMENTOS PARA QUANTIFICAÇÃO ESPECTROFOTOMÉTRICA DE FLAVONOIDES TOTAIS EM FOLHAS DE Bauhinia forficata LINK}

Graziella Silvestre Marques, Rebeka Priscila Maranhão Monteiro, Waleska de Figueirêdo Leão, Magaly Andreza Marques Lyra, Monize Santos Peixoto, Pedro José Rolim-Neto, Haroudo Sátiro Xavier e Luiz Alberto de Lira Soares*

Departamento de Ciências Farmacêuticas, Universidade Federal de Pernambuco, Rua Arthur de Sá, s/n, 50740-521 Recife - PE, Brasil

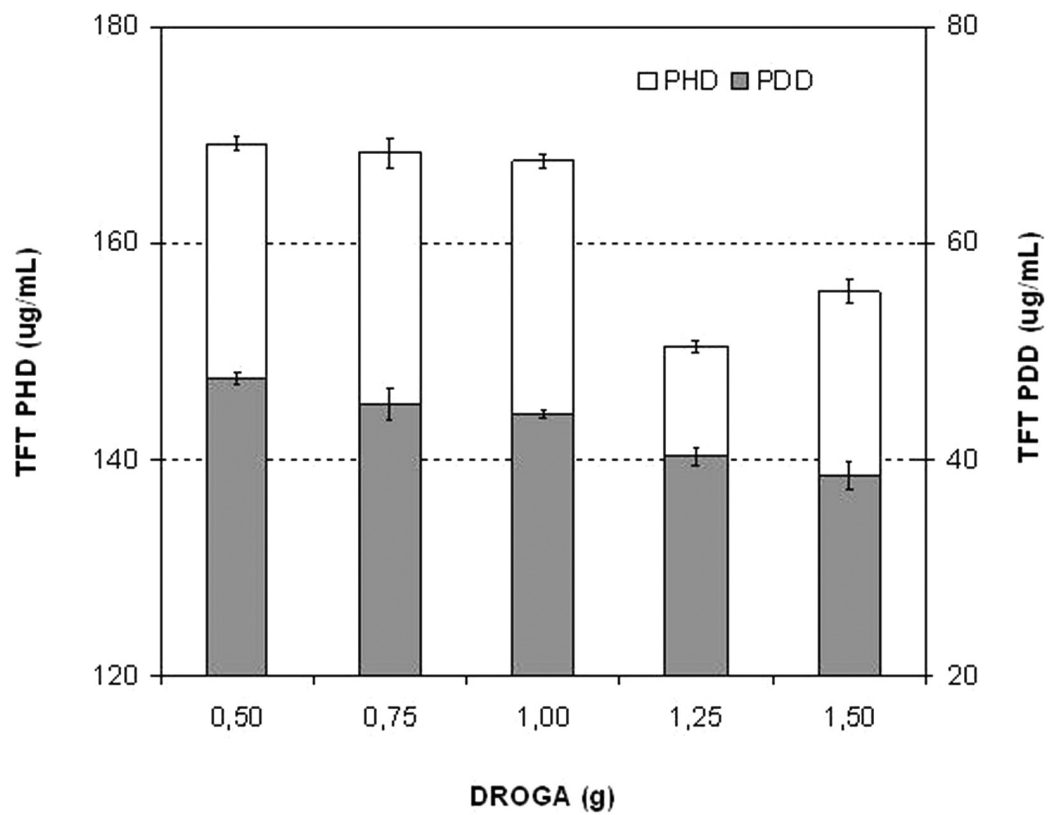

Figura 1S. Rendimento comparativo em g \% de flavonoides totais (TFT) para cada procedimento em função da quantidade de amostra

Tabela 1S. Resultados obtidos na análise da precisão intermediária da PDD e PHA

\begin{tabular}{lccccc}
\hline & & Dia $1(\mu \mathrm{g} / \mathrm{mL})$ & Dia 2 $(\mu \mathrm{g} / \mathrm{mL})$ & Analista & Dia \\
\hline PDD & Analista 1 & $41,16^{\mathrm{a}}$ & $41,95^{\mathrm{a}}$ & $\mathrm{F}_{\text {calculado }}=0,69$ & $\mathrm{~F}_{\text {calculado }}=0,85$ \\
& Analista 2 & $41,92^{\mathrm{a}}$ & $41,85^{\mathrm{a}}$ & $\mathrm{F}_{\text {tabelado }}=18,51$ & $\mathrm{~F}_{\text {tabelado }}=18,51$ \\
PHA & Analista 1 & $164,49^{\mathrm{a}}$ & $158,36^{\mathrm{a}}$ & $\mathrm{F}_{\text {calculado }}=1,36$ & $\mathrm{~F}_{\text {calculado }}=5,13$ \\
& Analista 2 & $163,49^{\mathrm{a}}$ & $161,70^{\mathrm{a}}$ & $\mathrm{F}_{\text {tabelado }}=18,51$ & $\mathrm{~F}_{\text {tabelado }}=18,51$ \\
\hline
\end{tabular}

PDD - Procedimento por diluição direta; PHA- Procedimento por hidrólise ácida; ${ }^{2}$ Resultado expresso em quercetina ( $\mu$ g de flavonoide/mL de extrato).

Tabela 2S. Resultados obtidos no teste de recuperação da PDD e PHA

\begin{tabular}{lcccc}
\hline & $\begin{array}{c}\text { Amostra } \\
(\mathrm{mg} / \mathrm{mL})\end{array}$ & $\begin{array}{c}\text { Valor teórico } \\
(\mu \mathrm{g} / \mathrm{mL})\end{array}$ & $\begin{array}{c}\text { Valor experimental }(\mu \mathrm{g} / \mathrm{mL}) \text { Média } \\
\pm \mathrm{dp}\end{array}$ & $\begin{array}{c}\text { Recuperação } \\
(\%)\end{array}$ \\
\hline PDD & 2,944 & $44,37^{\mathrm{a}}$ & $43,78^{\mathrm{a}} \pm 1,43$ & 98,66 \\
& 3,088 & $45,93^{\mathrm{a}}$ & $46,38^{\mathrm{a}} \pm 2,33$ & 100,99 \\
& 3,232 & $48,54^{\mathrm{a}}$ & $49,51^{\mathrm{a}} \pm 0,75$ & 101,99 \\
PHA & $171,11^{\mathrm{a}}$ & $174,30^{\mathrm{a}} \pm 0,41$ & 101,85 \\
& 0,6848 & $182,31^{\mathrm{a}}$ & $182,54^{\mathrm{a}} \pm 2,15$ & 100,12 \\
& 0,7296 & $193,50^{\mathrm{a}}$ & $192,35^{\mathrm{a}} \pm 4,15$ & 99,4 \\
\hline
\end{tabular}

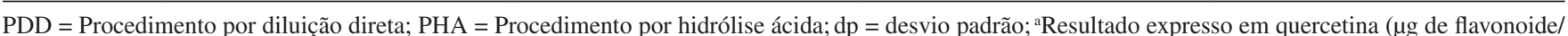
$\mathrm{mL}$ de extrato).

*e-mail: phtech@uol.com.br 
Tabela 3S. Resultados obtidos para análise da robustez da PDD

\begin{tabular}{|c|c|c|c|c|}
\hline Parâmetros & Variáveis & $\begin{array}{c}\text { Tempo } \\
\text { (h) }\end{array}$ & $\begin{array}{l}\text { TFT }(\mu \mathrm{g} / \mathrm{mL}) \\
\text { Média } \pm \mathrm{dp}\end{array}$ & $\mathrm{F}$ \\
\hline Luminosidade & $\begin{array}{l}\text { Ausência de luz } \\
\text { Presença de luz }\end{array}$ & $\begin{array}{l}--- \\
--- \\
\end{array}$ & $\begin{array}{l}40,26^{\mathrm{a}} \pm 0,01 \\
41,38^{\mathrm{a}} \pm 0,22 \\
\end{array}$ & $\begin{array}{l}\mathrm{F}_{\text {calculado }}=3,872 \\
\mathrm{~F}_{\text {tabelado }}=18,512\end{array}$ \\
\hline \multirow{2}{*}{ Estabilidade } & Ausência de luz & $\begin{array}{l}0 \\
3 \\
6 \\
\end{array}$ & $\begin{array}{l}40,27^{\mathrm{a}} \pm 0,42 \\
40,24^{\mathrm{a}} \pm 0,42 \\
41,03^{\mathrm{a}} \pm 0,13\end{array}$ & $\begin{array}{l}\mathrm{F}_{\text {calculado }}=4,823 \\
\mathrm{~F}_{\text {tabelado }}=5,143\end{array}$ \\
\hline & Presença de luz & $\begin{array}{l}0 \\
3 \\
6 \\
\end{array}$ & $\begin{array}{l}41,22^{\mathrm{a}} \pm 0,22 \\
41,54^{\mathrm{a}} \pm 0,50 \\
41,34^{\mathrm{a}} \pm 0,31\end{array}$ & $\begin{array}{l}\mathrm{F}_{\text {calculado }}=0,571 \\
\mathrm{~F}_{\text {tabelado }}=5,143\end{array}$ \\
\hline $\begin{array}{l}\text { Marca do solvente (álcool } \\
\text { etílico) }\end{array}$ & $\begin{array}{l}\text { Dinâmica }^{\circledR} \\
\text { Fmaia }^{\circledR}\end{array}$ & $\begin{array}{l}-- \\
--- \\
\end{array}$ & $\begin{array}{l}41,93^{\mathrm{a}} \pm 0,08 \\
41,39^{\mathrm{a}} \pm 0,37\end{array}$ & $\begin{array}{l}\mathrm{F}_{\text {calculado }}=5,727 \\
\mathrm{~F}_{\text {tabelado }}=7,708\end{array}$ \\
\hline
\end{tabular}

$\mathrm{dp}=$ desvio padrão; ${ }^{a}$ Resultado expresso em quercetina ( $\mu \mathrm{g}$ de flavonoide/mL de extrato).

Tabela 4S. Resultados obtidos para análise da robustez da PHA

\begin{tabular}{|c|c|c|c|c|}
\hline Parâmetros & Variáveis & $\begin{array}{c}\text { Tempo } \\
\text { (h) }\end{array}$ & $\begin{array}{l}\text { TFT }(\mu \mathrm{g} / \mathrm{mL}) \\
\text { Média } \pm \mathrm{dp}\end{array}$ & $\mathrm{F}$ \\
\hline Luminosidade & $\begin{array}{l}\text { Ausência de luz } \\
\text { Presença de luz }\end{array}$ & --- & $\begin{array}{l}163,71^{\mathrm{a}} \pm 1,93 \\
170,25^{\mathrm{a}} \pm 1,41\end{array}$ & $\begin{array}{l}\mathrm{F}_{\text {calculado }}=17,326 \\
\mathrm{~F}_{\text {tabelado }}=18,512\end{array}$ \\
\hline \multirow{2}{*}{ Estabilidade } & Ausência de luz & $\begin{array}{l}0 \\
3 \\
6 \\
\end{array}$ & $\begin{array}{l}162,49^{\mathrm{a}} \pm 1,76 \\
162,71^{\mathrm{a}} \pm 1,84 \\
165,94^{\mathrm{a}} \pm 0,51\end{array}$ & $\begin{aligned} \mathrm{F}_{\text {calculado }} & =4,961 \\
\mathrm{~F}_{\text {tabelado }} & =5,143\end{aligned}$ \\
\hline & Presença de luz & $\begin{array}{l}0 \\
3 \\
6 \\
\end{array}$ & $\begin{array}{l}162,49^{\mathrm{a}} \pm 1,76 \\
162,71^{\mathrm{a}} \pm 1,84 \\
165,94^{\mathrm{a}} \pm 0,51\end{array}$ & $\begin{aligned} \mathrm{F}_{\text {calculado }} & =2,664 \\
\mathrm{~F}_{\text {tabelado }} & =5,143\end{aligned}$ \\
\hline Marca do solvente (acetona) & $\begin{array}{l}\text { Dinâmica } \\
\text { Fmaia }^{\circledR}\end{array}$ & --- & $\begin{array}{l}161,04^{a} \pm 1,17 \\
161,93^{a} \pm 3,98\end{array}$ & $\begin{array}{l}\mathrm{F}_{\text {calculado }}=0,137 \\
\mathrm{~F}_{\text {tabelado }}=7,708\end{array}$ \\
\hline
\end{tabular}

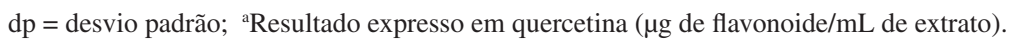

